\title{
Microestructura y propiedades mecánicas por microindentación y nanoindentación de un recubrimiento de circona estabilizada con itria (4,5\% en mol) elaborado por plasma atmosférico a partir de suspensiones
}

\author{
Microstructure and mechanical properties measured by microindentation and \\ nanoindentation of 4.5 YSZ coating elaborated by suspension plasma spraying
}

\author{
Andrés G. González H ${ }^{1,2 *} \quad$ Francy M. Hurtado ${ }^{1} \quad$ Esperanza López $^{1}$
}

Recibido 29 de febrero de 2016, aceptado 20 de junio de 2016

Received: February 29, 2016 Accepted: June 20, 2016

\begin{abstract}
RESUMEN
En este trabajo se analizó la relación entre la microestructura de un recubrimiento de 4,5\% en mol de circona estabilizada con itria (4.5YSZ) con sus propiedades mecánicas medidas por nanoindentación y microindentación. Los recubrimientos fueron elaborados por la técnica de proyección térmica por plasma atmosférico a partir de una suspensión de etanol y dos tipos de polvos ( 3 y $8 \%$ en mol de YSZ) con partículas de tamaño nanométrico. El análisis de la microestructura de la sección trasversal del recubrimiento de $4.5 \mathrm{YSZ}$ reveló una estructura bimodal, la que estaba compuesta de una zona con partículas semifundidas (ZS) y otra zona compuesta de lamelas con un alto grado de fusión (ZF). Los ensayos de nanoindentación mostraron una distribución Weibull bimodal para la dureza y monomodal para el módulo de elasticidad, lo que está relacionada con su estructura. Los ensayos microindentación (microdureza Vickers y módulo de elasticidad: E) presentaron una distribución Weibull monomodal. Estos resultados de microindentación estuvieron influenciados por el área de contacto de las zonas indentadas, ya que estas tomaban las dos zonas microestructurales (ZF y ZS) del recubrimiento de 4.5YSZ revelando una medición global de sus propiedades mecánicas.
\end{abstract}

Palabras clave: Proyección térmica por plasma, suspensiones de nanopartículas, recubrimiento de circona, nanoindentación, microindentación.

\section{ABSTRACT}

In this paper the microstructure of 4.5 mol\% Yttria-Stabilized-Zirconia (4.5YSZ) coating and its mechanical properties measured by nanoindentation and microhardness was analyzed. The coatings were elaborated by suspension plasma spraying (SPS) from a suspension of ethanol and two types of powders (3 and $8 \mathrm{~mol} \%$ YSZ) with nanosized particles. The analysis of the microstructure of the cross section of the $4.5 \mathrm{wt}$. YSZ coating revealed a bimodal structure, which was composed of a zone with semi-molten particles (ZS) and another zone consisting of lamellae with a high degree of fusion $(Z F)$. Nanoindentation tests showed a bimodal Weibull distribution for hardness and monomodal Weibull distribution for elastic modulus (E), which is related to its structure. Microhardness tests (hardness Vickers and E) had a monomodal Weibull distribution. These results of microindentation were influenced by the contact area of the indentation zones, as they took the two zones (ZF and ZS) of the microstructure of 4.5YSZ coating revealing global measurement of their mechanical properties.

Keywords: Suspension plasma spraying, coating, YSZ, nanoindentation, microindentation.

\footnotetext{
1 Universidad de Antioquia. Calle 67 N 53 -108. Medellín, Colombia. E-mail: francymhurtado@gmail.com; esperanza.lopez@udea.edu.co

2 Université de Limoges. Francia. E-mail: andresggonzalezh@gmail.com

* Autor de correspondencia
} 


\section{INTRODUCCIÓN}

La circona estabilizada con itria, conocida con sus siglas en inglés YSZ (Yttria-Stabilizated Zirconia) ha sido utilizada por décadas en sistemas de recubrimientos (multicapas) usados como barrera térmica [1-2] y una de sus aplicaciones más importantes a nivel industrial es ayudar a disminuir la temperatura de trabajo en alabes de las turbinas a gas [3-8]. Generalmente, el sistema de multicapas está compuesto de un recubrimiento cerámico de YSZ ("top coat") seguido de capa de anclaje o "bond coat", los que se aplican sobre una superaleación de níquel que generalmente corresponde a un Hastelloy, CMSX o Inconel $600,601,625,718$, entre las más utilizadas en los alabes de turbinas [9]. Durante el servicio de la turbina a gas, la que se somete a temperaturas entre 600 y $900{ }^{\circ} \mathrm{C}$, el recubrimiento disminuye aproximadamente la temperatura de trabajo de la aleación en $170{ }^{\circ} \mathrm{C}$ [10]. De esta manera, el recubrimiento logra mejorar la eficiencia y comportamiento en el proceso de la turbina, ya que incrementa el aislamiento térmico de sus componentes expuestos a estos ambientes agresivos. Industrialmente, el recubrimiento utilizado como barrera térmica (conocido en inglés como TBC, thermal barrier coating) generalmente es fabricado por la técnica de proyección térmica por plasma atmosférico conocido como APS (Atmospheric Plasma Spraying) o por la técnica de EB-PVD (Electro Beam-Physical Vapor Deposition).

La técnica de proyección térmica es un proceso para elaborar recubrimientos, donde la materia prima en forma de polvo o alambre es alimentado a una fuente de calor (antorcha o pistola) y luego el material en estado fundido o semifundido es proyectado a una alta velocidad para finalmente golpear el sustrato [4-6, 11]. Las partículas fundidas o semifundidas se van apilando sobre el sustrato para formar el recubrimiento. Así, la microestructura del recubrimiento en su sección transversal muestra una estructura laminar o "lamelas". En el proceso de APS, la materia prima en forma de polvo con tamaños entre 10-100 $\mu \mathrm{m}$ es alimentado a una antorcha de plasma, donde es fundida a una temperatura de aproximadamente $11.000{ }^{\circ} \mathrm{C}$ para ser proyectado a una velocidad entre $300-500 \mathrm{~m} / \mathrm{s}$ y finalmente forma el recubrimiento con espesor mínimo de $50 \mu \mathrm{m}$ y que puede llegar hasta algunos milímetros dependiendo del tiempo del proceso [12]. Esto se puede lograr entre 1 min y 10 min de duración del proceso y debido a esto, la técnica tiene una gran ventaja que es su alta rata de producción de un recubrimiento con respecto a otras técnicas como PVD y CVD.

En las últimas dos décadas se ha tenido un interés en fabricar recubrimientos nanoestructurados, ya que muestran arquitecturas diferentes y comportamientos interesantes, en cuanto a la mejora de sus propiedades mecánicas y térmicas en el caso de las barreras térmicas especialmente con el YSZ.

Actualmente, existen dos principales formas de obtener recubrimientos nanoestructurados mediante proyección térmica: (i) a partir de partículas nanométricas aglomeradas, o (ii) a partir de suspensiones de partículas nanométricas, técnica conocida como SPS (por sus siglas en inglés "Suspension Plasma Spraying") [13].

La técnica de SPS es una técnica relativamente nueva y los pioneros en este campo fueron Gitzhofer y Bouyer de la Universidad de Sherbrooke (Canadá) en 1997 ya que presentaron una patente con respecto a la inyección de una suspensión en un plasma de radio frecuencia [14]. Un esquema general del proceso a partir de suspensiones se muestra en la Figura 1. En los recubrimientos elaborados por esta técnica, la

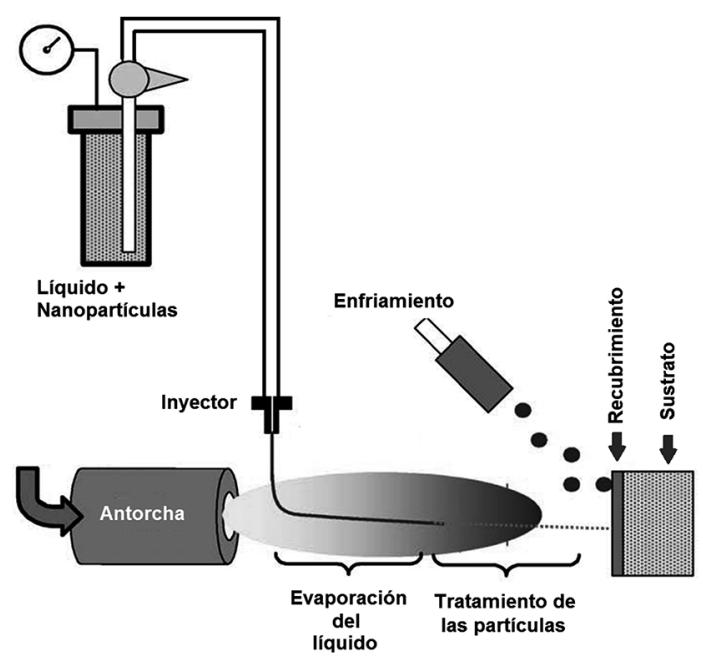

Figura 1. Esquema del proceso de proyección térmica para elaborar un recubrimiento a partir de una suspensión, SPS [16]. 
suspensión es inyectada preferiblemente a la zona de alta temperatura del jet de plasma o llama (ver Figura 1), donde la aglomeración de las partículas (generalmente cerámicas y nanométricas) puede ser controlada por acción mecánica (agitadores) o por acción química (mediante dispersantes o defloculantes) o ambos [15]. Posteriormente, cuando la suspensión interactúa con el jet de plasma o llama, esta se desintegra y luego se forman pequeñas gotas para evaporarse, así dejando que las partículas nanométricas entren en contacto con el plasma para fundirse o semifundirse, como se esquematiza en la Figura 1. Finalmente las partículas nanométricas fundidas o semifundidas son proyectadas a una alta velocidad hacia el sustrato para formar el recubrimiento caracterizado por su bajo espesor (normalmente entre 20-70 $\mu \mathrm{m}$ ) con respecto a las técnicas convencionales (desde $100 \mu \mathrm{m}$ hasta milímetros).

La diferencia entre técnica de SPS y la técnica de APS es principalmente que SPS utiliza un líquido portador de las partículas nanométricas (suspensión) en lugar de un gas de arrastre del polvo micrométrico como sucede en el proceso de APS [13]. Este cambio soluciona los problemas cuando se inyectan polvos nanométricos ya que, debido a su bajo flujo másico, generan obstrucciones de los sistemas de alimentación convencionales. De esta forma, la técnica SPS permite proyectar tamaños de partículas submicrométrico y nanométrico, lo que implica cierta ventaja con respecto a las técnicas convencionales, las cuales están limitadas únicamente a tamaños micrométricos [13].

Muchos trabajos de investigación sobre caracterización de las propiedades mecánicas de materiales y recubrimientos han sido publicados en los últimos años. Sin embargo, la cantidad de investigaciones en recubrimientos elaborados por SPS ha sido escasa [1718]. Entre los trabajos más importantes se encuentran [19-20], los que han estudiado la microestructura y las propiedades mecánicas por indentación de recubrimientos de YSZ, elaborados por SPS, a partir un polvo de tamaño nanométrico. En ambos estudios se encontró que los recubrimientos de YSZ, elaborados por SPS, presentan una estructura bimodal formada por partículas nanométricas rodeadas por lamelas completamente fundidas lo que causó una distribución bimodal de las propiedades (dureza y módulo de elasticidad).
Por otro lado, como consecuencia del bajo espesor obtenido en los recubrimientos de SPS, se ha encontrado que la técnica de nanoindentación es más factible y confiable que microindentación convencional para la caracterización mecánica de estos recubrimientos [17]. Sin embargo, en este trabajo se presenta un estudio de las propiedades mecánicas de un recubrimiento de 4.5YSZ, elaborado por SPS, medidas por nanoindentación y microindentación mostrando la diferencia en los resultados obtenidos en cada técnica. También se presenta un estudio de la relación entre la microestructura y las propiedades mecánicas del recubrimiento obtenido.

\section{METODOLOGÍA EXPERIMENTAL}

\section{Caracterización de la materia prima}

Dos polvos fueron seleccionados para elaborar el recubrimiento de 4.5YSZ: un polvo con $3 \%$ en mol de YSZ y otro con $8 \%$ en mol de YSZ. Su caracterización morfológica fue realizada mediante un microscopio electrónico de barrido de emisión de campo (FE-SEM: Field Emission Scanning Electron Microscopy) de marca JEOL 7400F. Los polvos nanométricos de composición 3\% mol YSZ y $8 \%$ mol YSZ fueron fabricados por la casa comercial UCM Advanced Ceramics GmbH de Alemania.

\section{Proceso de proyección térmica}

La suspensión para elaborar el recubrimiento fue preparada con etanol como solvente y un dispersante "Beycostat C213" el que fue utilizado para evitar la aglomeración de las partículas nanométricas. $\mathrm{La}$ suspensión utilizada en este estudio fue optimizada por Bacciochini [14] y Etchart-Salas [21], la que tiene un porcentaje de sólidos del $20 \%$ con un cantidad de dispersante del 2,1\% de la masa del polvo. Primero, el dispersante fue diluido en etanol mediante agitación magnética y con una sonda ultrasónica de alta potencia. Luego, los polvos fueron adicionados en pequeñas cantidades para evitar la formación de aglomerados. Los polvos de 3YSZ y 8YSZ fueron adicionados estequiométricamente $(62,7 \%$ en peso de polvo de 3 YSZ y $37,3 \%$ en peso de 8 YSZ) para lograr obtener la composición deseada en el recubrimiento (4,5\% en mol de YSZ). El material de los sustratos utilizados para la elaboración del recubrimiento fue acero inoxidable del tipo AISI/ SAE 304L con un diámetro de $25,4 \mathrm{~mm}$ y espesor de $9 \mathrm{~mm}$. Este tipo de sustratos generalmente es 
utilizado en procesos industriales a alta temperatura y equipos expuestos a ambientes corrosivos. La superficie a recubrir de estos sustratos fue pulida hasta lograr un acabado espejo.

Para elaborar el recubrimiento por proyección térmica por plasma a partir de suspensiones (SPS), se utilizó una antorcha convencional monocatódica tipo F4-MB (Wolhen, Suiza) con el sistema Multicoat de Sulzer Metco ${ }^{\circledR}$ y un sistema de inyección de la suspensión, el que estaba montado en el laboratorio SPCTS de la Universidad de Limoges (Francia). El inyector utilizado para llevar la suspensión al centro del plasma tenía un diámetro de $150 \mu \mathrm{m}$ y este es ajustado manualmente antes del proceso, de tal manera que la fragmentación de la suspensión se llevara a cabo dentro del jet de plasma, tal y como se muestra en la Figura 2.

Ensayos preliminares realizados permitieron definir los parámetros de elaboración de los recubrimientos por SPS los que fueron: (i) distancia de $3 \mathrm{~cm}$ entre la punta de la antorcha y el sustrato; (ii) intensidad de corriente en la antorcha de $500 \mathrm{~A}$; (iii) gases plasmagénicos de Ar-He con un flujo de 40-20 slpm respectivamente; (iv) temperatura de precalentamiento del sustrato $200^{\circ} \mathrm{C}$ y (v) tiempo total de aplicación de 4 min sin superar los $400{ }^{\circ} \mathrm{C}$.

\section{Caracterización morfológica, química y mecánica del recubrimiento}

La muestra del recubrimiento de $4.5 \mathrm{YSZ}$ obtenido por SPS para la caracterización morfológica y mecánica fue pulida teniendo en cuenta la norma

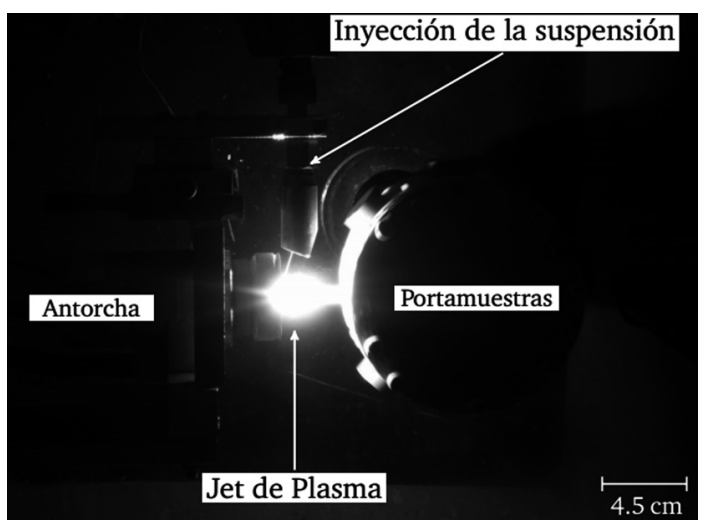

Figura 2. Elaboración del recubrimiento por plasma atmosférico a partir de una suspensión (SPS).
ASTM E1920-03 [22]. El análisis de la sección transversal pulida del recubrimiento y la morfología de las indentaciones producto de los ensayos de microindentación y nanoindentación fueron analizadas mediante un microscopio electrónico de barrido de la marca JEOL JSM-6490LV. Para verificar la composición química del recubrimiento se utilizó la técnica de fluorescencia de rayos $\mathrm{X}$ (FRX) por longitud de onda dispersiva, utilizando un espectrómetro de OPTIM'X de Thermo Scientific. Las fases presentes en el recubrimiento fueron determinadas a partir de un espectro de difracción de rayos $X$ (DRX), el que fue obtenido por un difractómetro Siemens D5000 con una fuente de cobre ( $\mathrm{Cu} \mathrm{K}$ - $\alpha$ de $\lambda=0,154 \mathrm{~nm}$ ) con una velocidad de barrido de $0,020^{\circ}$ por paso. El espectro fue analizado con ayuda del software X'Pert HighScore.

Los ensayos de nanoindentación se realizaron en un equipo Nano Indenter XP de MTS Systems Corporation. Se hicieron 12 indentaciones en línea recta espaciadas $40 \mu \mathrm{m}$ cada una, realizadas en la mitad de la sección transversal pulida del recubrimiento, evitando así el efecto del sustrato y el borde del recubrimiento. Las indentaciones Berkovich fueron distribuidas en tres regiones a lo largo de la sección transversal, realizando cuatro indentaciones en cada región. Las cargas preliminarmente fueron estudiadas en un rango entre 100 a $500 \mathrm{mN}$. De este rango de cargas se seleccionó experimentalmente una carga de $100 \mathrm{mN}(10 \mathrm{~g})$ para realizar las medidas de dureza y módulo de elasticidad, utilizando el modo continuo stiffness measurement (CSM) que permite obtener una medida continua de los datos. La dureza (H) y el módulo de elasticidad (E) se calcularon a partir de los datos de la curva carga-descarga P-h obtenida con un indentador Berkovich mediante el método de Oliver y Pharr [23]. Los valores de dureza y módulo de elasticidad fueron calculados directamente por el software del equipo.

Los ensayos de microindentación se realizaron en un equipo Shimadzu serie G20, bajo la norma ASTM C1327-08 [24] para indentaciones Vickers y bajo la norma ASTM C1326-03 [24] para indentaciones Knoop [25]. Se utilizó un indentador Vickers para calcular la dureza Vickers (HV) variando la carga entre 25 y $100 \mathrm{~g}$ y se hicieron 20 medidas por cada carga en la sección transversal pulida del recubrimiento. Esto se hizo para encontrar la carga crítica a la que la recuperación elástica no 
es tan pequeña ni tan grande, que se considera que no hay una sobreestimación ni una subestimación de la microdureza. Se encontró una carga crítica de $60 \mathrm{~g}$ para el recubrimiento estudiado, la que se aplicó durante $15 \mathrm{~s}$ espaciadas $40 \mu \mathrm{m}$ una de la otra, para evitar la influencia de las indentaciones vecinas. Se seleccionaron 13 huellas aceptables por la norma de las 20 realizadas. Por otro lado, se utilizó un indentador Knoop para hallar la dureza Knoop (HK), la que fue utilizada para calcular el módulo de elasticidad (E), utilizando el método de Marshall [26]. Para el ensayo se hicieron 20 indentaciones Knoop a una carga de 125 g. La carga fue aplicada durante $15 \mathrm{~s}$ y las huellas fueron distribuidas en toda la muestra a una distancia de aproximadamente $150 \mu \mathrm{m}$ una de la otra, para evitar la influencia de las indentaciones vecinas. Se seleccionaron 13 huellas aceptables por la norma de las 20 realizadas en la sección transversal del recubrimiento.

Los datos del módulo de elasticidad y dureza hallados por las dos técnicas de indentación, fueron analizados mediante la distribución estadística de Weibull. Esta distribución es un indicador de la variabilidad de la resistencia de los materiales, debido a una distribución de tamaños de las imperfecciones [27] y cuanto mayor es su valor, menor es la variabilidad de la propiedad medida. Las curvas se construyeron en el software OriginPro 8.5 y a partir de este se obtuvo el módulo de Weibull, que representa el grado de variabilidad de cada una de las propiedades halladas. Además el valor de la dureza y del módulo de elasticidad fue hallado gráficamente como el resultado del percentil 50 .

\section{RESULTADOS Y ANÁLISIS}

\section{Morfología de la materia prima}

La morfología del polvo de circona estabilizada con $3 \%$ en mol de itria y $8 \%$ en mol de itria es mostrada en la Figura 3.

Los polvos presentan partículas con una forma irregular y angular, y están muy relacionados con su método de fabricación, el que fue mediante una molienda de alta energía. Las micrografías muestran una magnificación de 30.000 aumentos, donde se aprecia que las partículas de los polvos de tamaño nanométrico se encuentran ligeramente aglomeradas y además se puede observar que
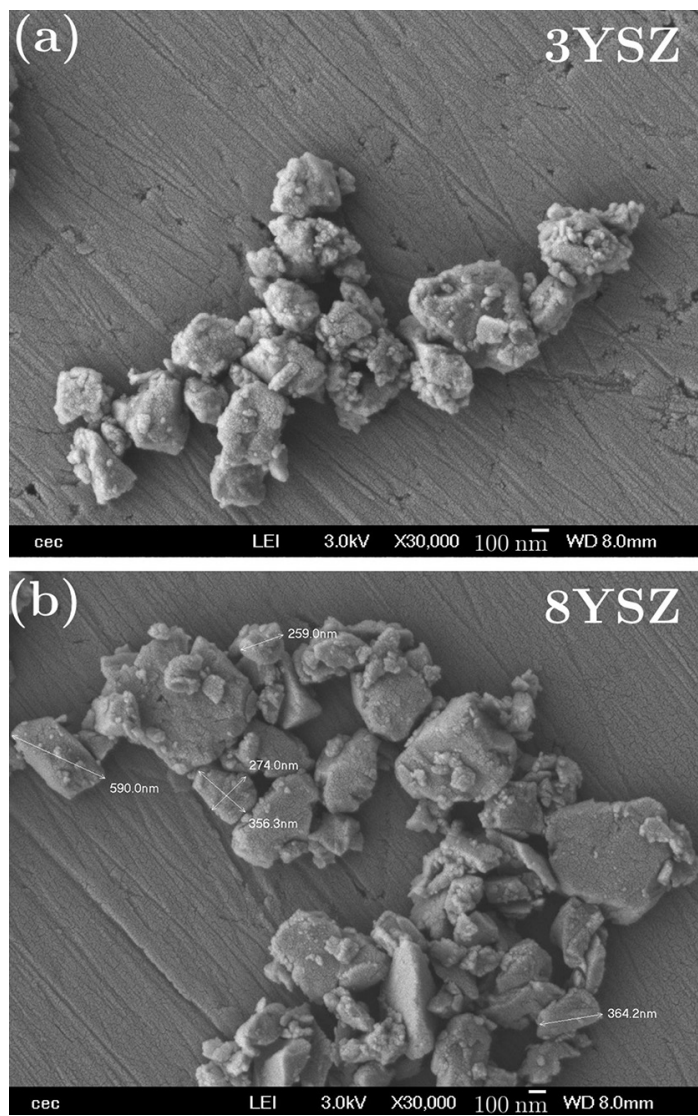

Figura 3. Morfología de los polvos de tamaño nanométrico de (a) 3YSZ y (b) 8YSZ por FE-SEM a 30000X.

el tamaño de partícula de los polvos 3YSZ y 8 YSZ están en un rango entre 100 y 900 nm aproximadamente.

De acuerdo a las fases analizadas por Difracción de Rayos X (DRX), el polvo de 3YSZ tiene las fases de circona tetragonal y monoclínica, lo que corresponde precisamente con su composición química, lo que corrobora que está compuesto de circona parcialmente estabilizada. Por otro lado, el polvo 8YSZ tiene como única fase a la circona tetragonal, lo que también corrobora que corresponde con su composición química al ser un polvo de circona totalmente estabilizada. Es importante resaltar que los dos polvos fueron mezclados para obtener la composición química más común encontrada en las barreras térmicas y además a nivel comercial es difícil la consecución de polvos nanométricos de dicha composición química $(4,5 \% \mathrm{~mol})$. 


\section{Análisis estructural del recubrimiento}

La sección transversal del recubrimiento de 4,5\% en mol de YSZ obtenido por SPS se muestra en la Figura 4. Se observa que el recubrimiento tiene una estructura homogénea en general (ver Figura 4a y Figura $4 b$ ), con presencia de poros y sin grietas
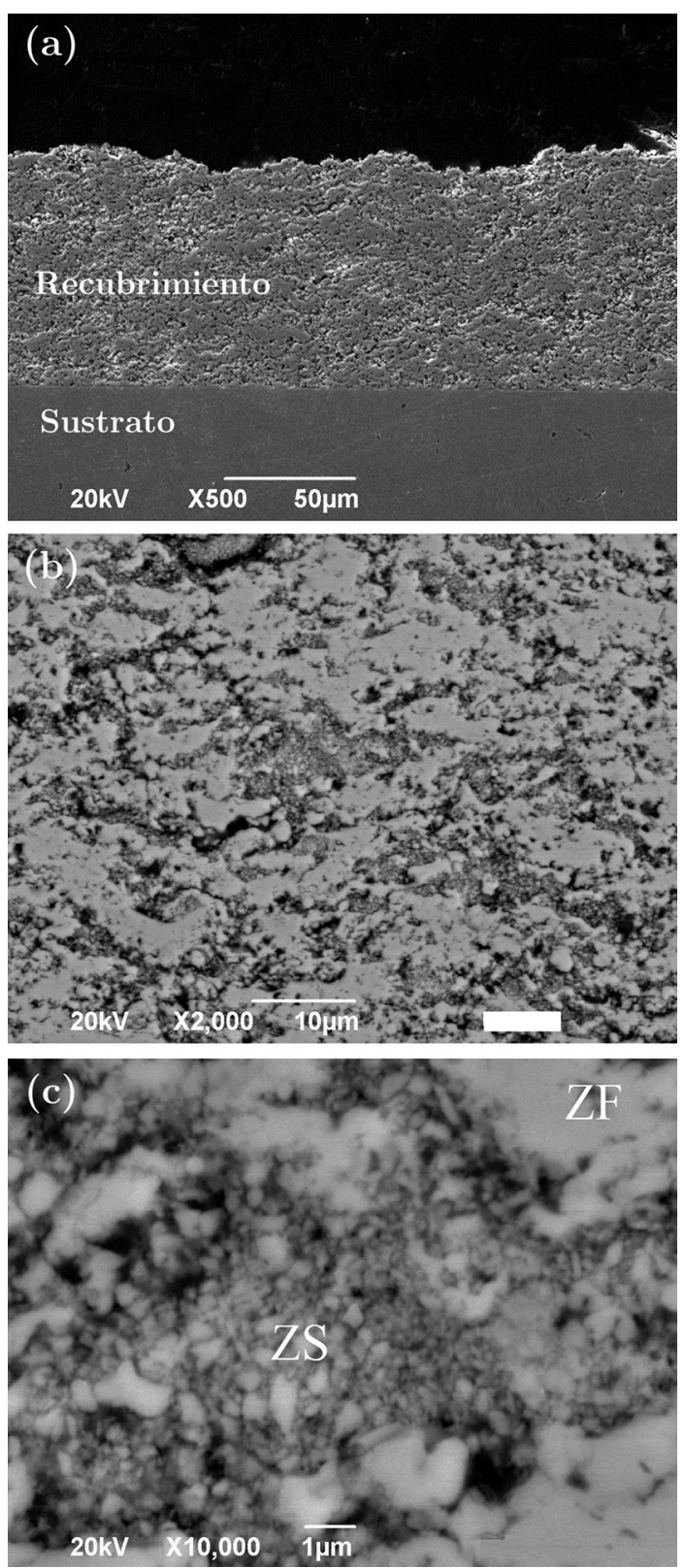

Figura 4. Sección transversal del recubrimiento de 4.5YSZ: (a) Vista general, (b) Ampliación de la capa cerámica y (c) Zona semifundida (ZS) y Zona fundida (ZF). apreciables. Además, el recubrimiento obtenido exhibió un espesor promedio de $70 \pm 2 \mu \mathrm{m}$ y su porosidad fue del $9,6 \%$, la que fue medida a partir de 10 imágenes MEB analizadas con el Software ImageJ. En la Figura 4c se muestra una ampliación a 10.000 aumentos de la capa de YSZ del recubrimiento, la que tiene una estructura compuesta por dos zonas: (i) una zona densa con partículas nanométricas semifundidas (ZS) y (ii) lamelas con mayor grado de fusión (ZF) que se encuentran regularmente distribuidas, como se muestra en la Figura 4b. De esta forma, se puede decir que el recubrimiento obtenido es nanoestructurado.

El resultado del análisis químico realizado mediante FRX al recubrimiento de 4,5\% en mol de YSZ se muestra en la Tabla 1. En la tabla se puede observar que la composición química (\% en peso) analizada por FRX corresponde a la relación en moles entre la circona $\left(\mathrm{ZrO}_{2}\right)$ y la itria $\left(\mathrm{Y}_{2} \mathrm{O}_{3}\right)$ del $4,5 \%$ en mol. $\mathrm{El} \mathrm{HfO}_{2}$ es un compuesto proveniente de la materia prima, especialmente de su método de fabricación.

Tabla 1. Resultado del análisis químico por FRX realizado al recubrimiento de $4.5 \mathrm{YSZ}$ elaborados por SPS.

\begin{tabular}{|c|c|}
\hline Compuesto & \% en peso \\
\hline $\mathrm{ZrO}_{2}$ & 90,39 \\
\hline $\mathrm{Y}_{2} \mathrm{O}_{3}$ & 7,69 \\
\hline $\mathrm{HfO}_{2}$ & 1,47 \\
\hline $\mathrm{Al}_{2} \mathrm{O}_{3}$ & 0,45 \\
\hline
\end{tabular}

El espectro de DRX del recubrimiento de 4.5YSZ elaborado por SPS se muestra en la Figura 5. El recubrimiento muestra una única fase compuesta de circona tetragonal con fórmula química $\mathrm{t}-\mathrm{Zr}_{0,82} \mathrm{Y}_{0,18} \mathrm{O}_{1,91}$.

A pesar que el recubrimiento fue fabricado a partir de una suspensión con una gran cantidad del polvo de 3 YSZ (62,7\% en peso), el recubrimiento no presentaba la fase circona monoclínica $\left(\mathrm{m}-\mathrm{ZrO}_{2}\right)$ proveniente de su materia prima (ver Figura 5). Esto quiere decir que durante el proceso de SPS, la composición química de mezcla de la suspensión 4.5YSZ, la temperatura del plasma y la rápida solidificación en el proceso, originó que se transformara la cantidad de la circona monoclínica 


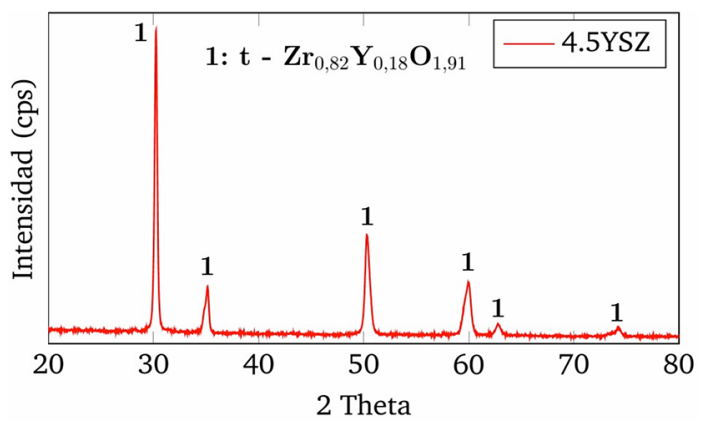

Figura 5. Espectro de DRX del recubrimiento.

que existía en su materia prima (3YSZ) en la única fase encontrada del recubrimiento, la que fue circona tetragonal ( $\left.\mathrm{t}-\mathrm{Zr}_{0,82} \mathrm{Y}_{0,18} \mathrm{O}_{1,91}\right)$. Por tanto, el recubrimiento tiene una circona totalmente estabilizada y es beneficioso ya que la fase tetragonal tiene mejores propiedades mecánicas que la fase monoclínica de la circona.

\section{Caracterización mecánica del recubrimiento por nanoindentación}

Estudios anteriores demuestran que las propiedades mecánicas de recubrimientos elaborados por SPS evaluadas por nanoindentación dependen, en gran medida, de la localización de la indentación y de la presencia de poros y grietas, además de la estructura del material en la vecindad de la indentación. Por ejemplo, Nohava, Mušálek, Matějičck y Vilémová [28] han observado fenómenos similares en recubrimientos elaborados por proyección térmica, concluyendo que la desviación estándar muestra que el material es altamente heterogéneo y que la medición de las propiedades mecánicas depende resistentemente de la porosidad del recubrimiento y la ubicación de la indentación. Latka, Chicot, Cattini, Pawlowski y Ambroziak [29] señalan en sus estudios que la desviación estándar de las propiedades mecánicas decrece cuando hay una disminución en el tamaño del defecto en proporción con el tamaño de la indentación.

La Figura 6 muestra el patrón de la curva cargadescarga (P-h) y el modelo estadístico de Weibull para la dureza y el módulo de elasticidad medido por nanoindentación del recubrimiento estudiado.

En la curva P-h (Figura 6a) no se observan saltos o cambios en la curva de carga, lo que indica que el recubrimiento está libre de poros y grietas
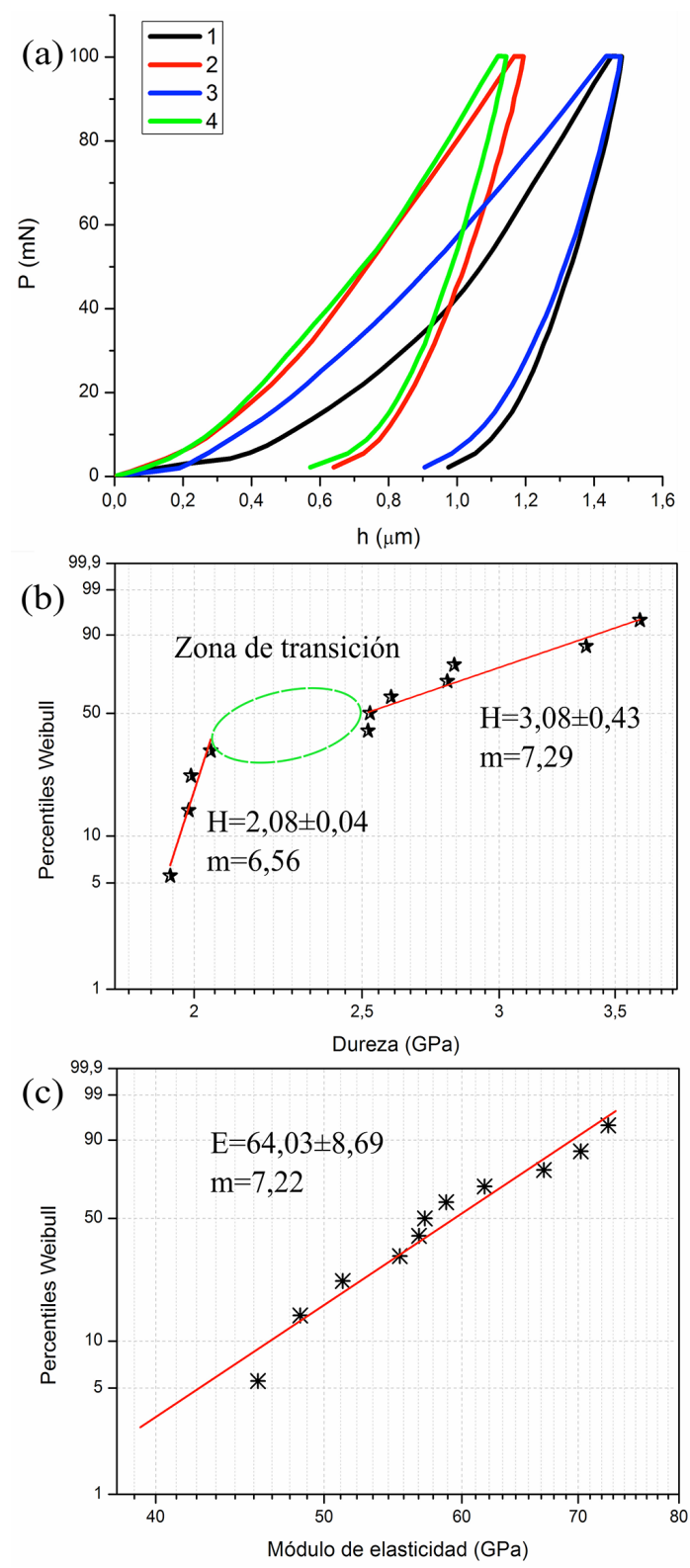

Figura 6. Sección transversal del recubrimiento (a) Patrón de la curva carga-descarga P-h para las huellas de la 1-4. Modelo estadístico Weibull para (b) Dureza y (c) Módulo de elasticidad.

perceptibles para la técnica de nanoindentación, acorde a la literatura consultada. Además, se observa que hubo una disminución en la profundidad de penetración a carga máxima de 0,95 a $0,60 \mu \mathrm{m}$, lo que fue influenciado por la presencia de zonas con diferente dureza en el recubrimiento. Esto se 
corrobora con los datos de la gráfica de la Figura $6 \mathrm{~b}$, donde se muestra la distribución estadística de Weibull de la dureza del recubrimiento. Se observa que los valores de dureza tienen una distribución bimodal y de acuerdo a la observación por MEB de las huellas, se pudo establecer que el valor de $3,1 \pm 0,43 \mathrm{GPa}$ de dureza estaba relacionada con la zona fundida (ZF, ver Figura 4c) del recubrimiento y que el valor de 2,1 $\pm 0,04 \mathrm{GPa}$ correspondía a zonas nanoestructuradas compuestas de partículas semifundidas (ZS). Además de las dos zonas mencionadas, se observa una tercera que se refiere a una zona de transición, en la que el valor de la dureza es intermedio, como se muestra en la Figura 6 b.

Por otro lado, se encontró que el módulo de elasticidad presenta un comportamiento monomodal con un valor de 64,03 $\pm 8,69 \mathrm{GPa}$ (Figura 6c), el que es inferior al valor de módulo de elasticidad reportado en la literatura $[17,30,31]$ para recubrimientos de YSZ elaborados por SPS. Este comportamiento puede deberse a que la ZF no está completamente fundida como se esperaba y esta disminución en su grado de fusión pudo atribuirse al corto tiempo de residencia de las partículas nanométricas en el plasma generando un recubrimiento con gran cantidad de zonas con partículas nanométricas semifundidas. Por esto, la diferencia en el grado de fusión entre las zonas (ZS y ZF) que componen el recubrimiento no es suficiente para que exista una diferencia notable en el módulo de elasticidad del recubrimiento obtenido.

Dos micrografías de huellas representativas en los ensayos de nanoindentación realizadas en el recubrimiento se muestran en la Figura 7. Se evidencia la existencia de una notable diferencia en el tamaño de las huellas Berkovich, lo que demuestra la presencia de las dos zonas con diferente dureza influenciadas por los dos tipos de estructuras encontradas en el recubrimiento (ZS y ZF). La zona que tiene partículas semifundidas (ver Figura 7a) es mucho más blanda que la zona con partículas de mayor grado de fusión (ver Figura 7b), como se puede ver en el valor de la dureza de la Figura 7. Es importante resaltar que las indentaciones Berkovich fueron realizadas en las mismas condiciones.

\section{Caracterización mecánica del recubrimiento por microindentación}

El modelo estadístico de Weibull de microdureza Vickers (HV) y módulo de elasticidad (E) se muestra
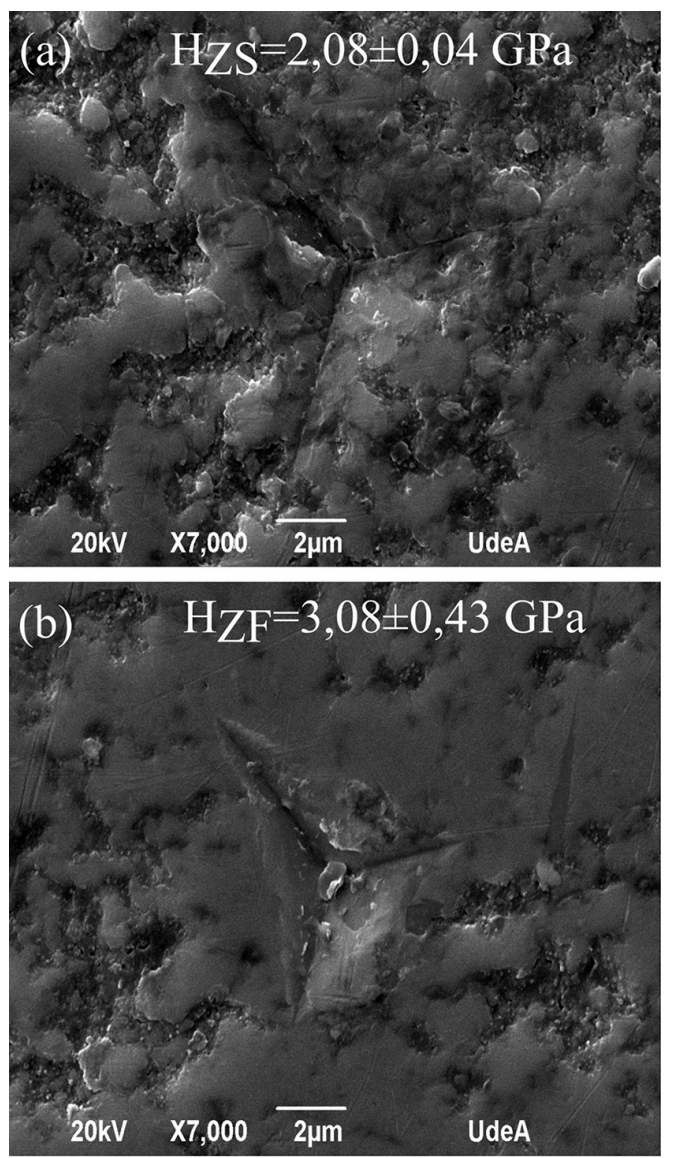

Figura 7. Huellas de nanoindentación ubicadas en la (a) Zona semifundida y (b) Zona fundida del recubrimiento de 4.5YSZ.

en la Figura 8. Una distribución estadística de Weibull para la dureza del recubrimiento (Figura 8a) tiene un comportamiento monomodal con un valor de HV de 3,41 \pm 0,39 GPa. Además, el mismo comportamiento (monomodal) lo presenta el módulo de elasticidad con un valor de 96,71 \pm 9,26 GPa. Es de resaltar que este comportamiento se debe principalmente a que la indentación Vickers, por su tamaño, abarcan las dos zonas de recubrimiento (fundida y semifundida), lo que es difícil encontrar dos comportamientos diferentes en las propiedades mecánicas evaluadas por microindentación en relación con su microestructura.

Una consideración adicional que se debe tener en cuenta para entender la forma de las huellas obtenidas por microindentación en el tipo de recubrimiento estudiado (estructura bimodal), es el fenómeno 
(a)

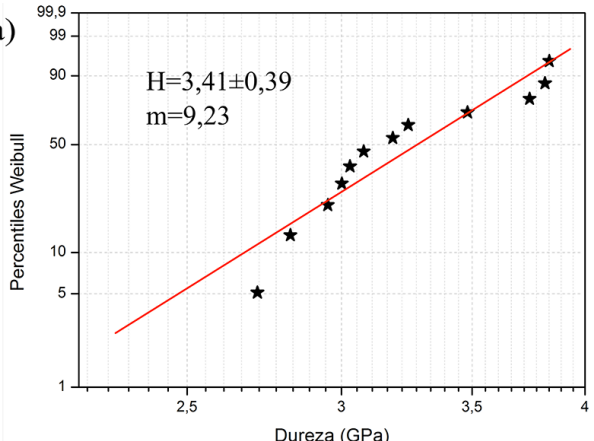

(b)

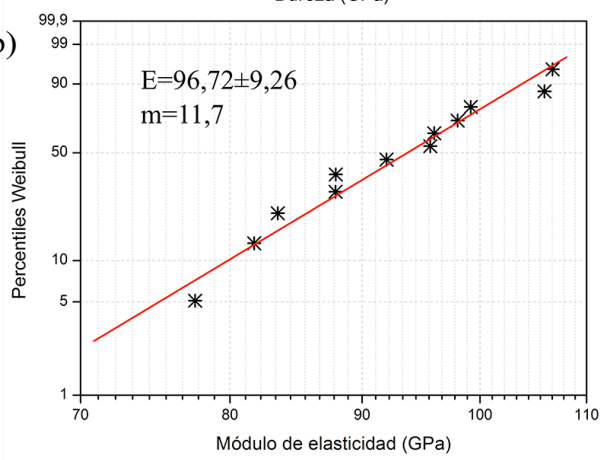

Figura 8. Modelo estadístico Weibull para (a) Microdureza HV y el (b) Módulo de elasticidad E.

de apilamiento que ocurre en la zona adyacente al área indentada. Cuando la zona adyacente al área indentada es más blanda (zona semifundida), facilita la penetración del indentador, lo que hace que las aristas de la huella obtenida adquieran cierta curvatura que le proporciona desprendimientos en las áreas de mayor concentración de esfuerzos, tal y como se muestra en la Figura 9.

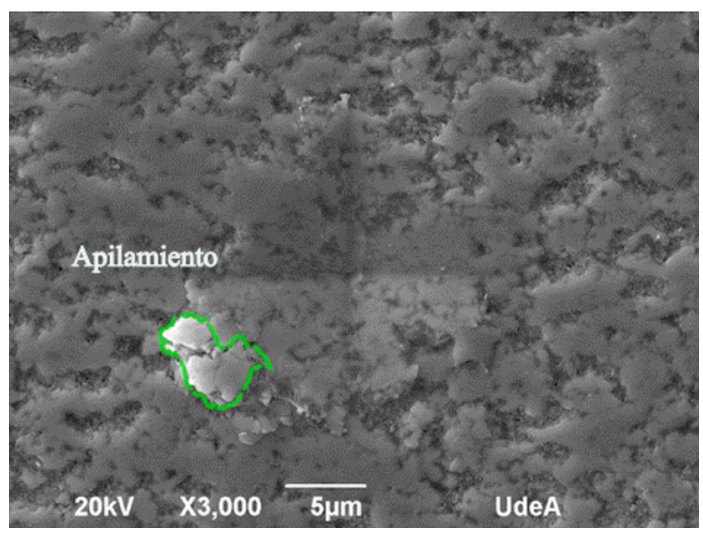

Figura 9. Huella de microindentación con desprendimiento del material del recubrimiento.

\section{CONCLUSIONES}

El recubrimiento de 4.5YSZ fue elaborado por la técnica de proyección térmica por plasma atmosférico a partir de una suspensión (SPS), la que estaba compuesta de una mezcla de polvos de 3YSZ y 8YSZ con partículas de tamaño nanométrico (100-900 nm). Este recubrimiento obtenido estaba compuesto de una estructura bimodal, la que se distinguía por zonas fundidas (ZF) y zonas nanoestructuradas proveniente de partículas semifundidas (ZS). Los análisis de DRX demostraron la presencia de circona con una única fase tetragonal, es decir, el recubrimiento presentó circona totalmente estabilizada, lo cual es beneficioso para aplicaciones a alta temperatura como en barreras térmicas. Los análisis de FRX corroboraron la composición química del recubrimiento elaborado (4,5\% en mol YSZ).

A partir de la distribución estadística de Weibull y de los valores de las propiedades mecánicas medidas por microindentación y nanoindentación, se encontró que la técnica de microindentación es menos sensible a la presencia de características y defectos como: grietas, poros y zonas nanoestructuradas, que la técnica de nanoindentación, lo cual se explica porque los ensayos de microindentación comprenden tanto la zona semifundida (ZS) como la más fundida (ZF) y a su vez los ensayos de nanoindentación están en la capacidad de discriminar entre las diferentes regiones estructurales debido a que se utilizan cargas muy pequeñas, además de la forma del indentador y el tamaño de la huella generada.

Adicionalmente, se comprobó que la técnica de microindentación es adecuada para caracterizar recubrimientos elaborados por SPS $(>70 \mu \mathrm{m}$ de espesor), siempre y cuando se tengan las consideraciones pertinentes. Es importante resaltar que la técnica de microindentación es una herramienta eficaz para medir las propiedades mecánicas globales del recubrimiento por SPS, mientras que la técnica de nanoindentación (especialmente en dureza) puede distinguir las propiedades mecánicas de las diferentes zonas nanoestructuradas de un recubrimiento elaborado por SPS (ZF y ZS).

\section{AGRADECIMIENTOS}

Los autores agradecen a la Universidad de Antioquia (Colombia) por su financiación mediante los 
proyectos CODI MDC10-01-04, PR14-2-05 y al programa "Estrategia de Sostenibilidad 2014-2015 de la Universidad de Antioquia". El autor Andrés González agradece a Colciencias y Colfuturo por el apoyo financiero en sus estudios de doctorado realizados en la Universidad de Antioquia y en la Universidad de Limoges (Francia).

\section{REFERENCIAS}

[1] X.Q. Cao, R. Vassen and D. Stoever. "Ceramic materials for thermal barrier coatings". Journal of the European Ceramic Society. Vol. $24 \mathrm{~N}^{\circ}$ 1, pp. 1-10. 2004. ISSN: 09552219 10.1016/S0955-2219(03)00129-8.

[2] J.D. Osorio, A. Lopera-Valle, A. Toro and J.P. Hernández-Ortiz. "Phase transformations in air plasma-sprayed yttria-stabilized zirconia thermal barrier coating". Dyna. Vol. 81 No 185, pp. 13-18. 2014. URL: http://dx.doi. org/10.15446/dyna.v81n185.33409.

[3] P. Carpio, E. Bannier, A. Borrell, M.D. Salvador y E. Sánchez. "Influencia de los parámetros de proyección por plasma atmosférico en recubrimientos de YSZ obtenidos a partir de polvos micro y nanoestructurados". Boletín de la Sociedad Española de Cerámica y Vidrio. Vol. 53 $\mathrm{N}^{\circ}$ 4, pp. 162-170. Septiembre 2014. ISSN: 2173-0431. DOI: 10.3989/cyv.202014.

[4] A. Salazar, J. Gómez-García, V. Utrilla y P. Poza. "Degradación de recubrimientos de $\mathrm{ZrO}_{2}-\mathrm{CaO} / \mathrm{NiAlMo}$ por oxidación isoterma". Boletín de la Sociedad Española de Cerámica y Vidrio. Vol. 45 No 6, pp. 389-395. 2006.

[5] A.G. González, E. López, A. Tamayo, E. Restrepo and F. Hernández. "Análisis de la microestructura y de las fases de recubrimientos de zirconia-alumina elaborados por proyección térmica”. Dyna. Vol. 77 Nº 162, pp. 151-160. 2009. ISSN: 00127353.

[6] M.V. Utrilla, P. Poza, J. Gómez-García, M.D. López, C.J. Múnez y M. Pichel. "Degradación de barreras térmicas por sales fundidas". Boletín de la Sociedad Española de Cerámica y Vidrio. Vol. 47 № 2, pp. 81-88. 2008. ISSN: 03663175.

[7] J. Gómez-García, A. Salazar, C.J. Múnez, V. Utrilla y P. Poza. "Análisis de la degradación de recubrimientos de barrera térmica por espectroscopia de impedancia electroquímica". Boletín de la Sociedad Española de Cerámica y Vidrio. Vol. 46 No 5, pp. 232-239. 2007.

[8] R. Ghasemi, R. Shoja-Razavi, R. Mozafarinia and H. Jamali. "Comparison of microstructure and mechanical properties of plasma-sprayed nanostructured and conventional yttria stabilized zirconia thermal barrier coatings". Ceramics International. Vol. $39 \mathrm{~N}^{\circ}$ 8, pp. 88058813. December, 2013. ISSN: 02728842. DOI: 10.1016/j.ceramint.2013.04.068.

[9] J.D. Osorio, J.P. Hernández-Ortiz, and A. Toro. "Microstructure characterization of thermal barrier coating systems after controlled exposure to a high temperature". Ceramics International. Vol. 40. $\mathrm{N}^{\circ} 3$. pp. 4663-4671. 2014. ISSN: 02728842 10.1016/j.ceramint.2013.09.007.

[10] Y. Zhao, D. Li, X. Zhong, H. Zhao, L. Wang, F. Shao, C. Liu and S. Tao. "Thermal shock behaviors of YSZ thick thermal barrier coatings fabricated by suspension and atmospheric plasma spraying". Surface and Coatings Technology. Vol. 249, pp. 48-55. 2014. ISSN: 02578972. DOI: 10.1016/j. surfcoat.2014.03.046.

[11] A. González, J. Henao, A.F. Díaz, E. López y F. Vargas. "Influencia de los parámetros de proyección térmica en la microestructura de los recubrimientos de circona-alúmina y circona-ceria usados como barreras térmicas". Revista Latinoamericana de Metalurgia y Materiales. Vol. 33 N $^{\circ}$ 2, pp. 272-281. 2013.

[12] R. Vaen, H. Kaner, G. Mauer and D. Stöver. "Suspension plasma spraying: Process characteristics and applications". Journal of Thermal Spray Technology. Vol. $19 \mathrm{~N}^{\mathrm{o}} 1-2$, pp. 219-225. 2010. ISSN: 10599630. DOI: 10.1007/s11666-009-9451-x.

[13] P. Fauchais, V. Rat, J.F. Coudert, R. Etchart-Salas and G. Montavon. "Operating parameters for suspension and solution plasma-spray coatings". Surface and Coatings Technology. Vol. 202 No $^{\circ} 18$, pp. 4309-4317. 2008. ISSN: 02578972. DOI: 10.1016/j. surfcoat.2008.04.003.

[14] A. Bacciochini. "Quantification de l'architecture poreuse de dépôts finement structurés (sub-micromètre - nanomètre) de zircone yttriée realisés par projection plasma de suspension”. Université de Limoges. 2010. 
[15] A.G. González, F. Hurtado, H. Ageorges, E. Gómez y F. Vargas. "Estudio de la estructura y las propiedades mecánicas en un recubrimiento de circona estabilizada con $8 \%$ en mol de itria elaborado por proyección térmica por plasma a partir de suspensiones". Máteria (Rio J.) [Online]. Vol. $21 \mathrm{~N}^{\mathrm{o}} 1$, pp. 41-52. 2016.

[16] K. Wittmann-Ténèze, K. Vallé, L. Bianchi, P. Belleville and N. Caron. "Nanostructured zirconia coatings processed by PROSOL deposition". Surface and Coatings Technology. Vol. 202 No $^{\circ} 18$, pp. 4349-4354. 2008. ISSN: 02578972. DOI: 10.1016/j. surfcoat.2008.04.008.

[17] P. Carpio, E. Rayón, L. Pawłowski, A. Cattini, R. Benavente, E. Bannier, M. D. D. Salvador and E. Sánchez. "Microstructure and indentation mechanical properties of YSZ nanostructured coatings obtained by suspension plasma spraying". Surface and Coatings Technology. Vol. 220, pp. 237243. April, 2013. ISSN: 02578972. DOI: 10.1016/j.surfcoat.2012.09.047.

[18] R. Vert, D. Chicot, C. Dublanche-Tixier, E. Meillot, A. Vardelle, and G. Mariaux. "Adhesion of YSZ suspension plasma-sprayed coating on smooth and thin substrates". Surface and Coatings Technology. Vol. 205 No 4, pp. 999-1003. 2010. ISSN: 02578972. DOI: 10.1016/j.surfcoat.2010.07.090.

[19] L. Wang, X.H. Zhong, J.S. Yang, S.Y. Tao, W. Zhang, Y. Wang and X.G. Sun. "Finite element simulation of surface micro-indentation behavior of yttria stabilized zirconia thermal barrier coatings with microstructural characteristic of columnar grains and subgrains based on a nonlinear contact model". Computational Materials Science. Vol. 82, pp. 244-256. February, 2014. ISSN: 09270256 10.1016/j.commatsci.2013.09.034.

[20] S. Kozerski, L. Łatka, L. Pawlowski, F. Cernuschi, F. Petit, C. Pierlot, H. Podlesak, and J. P. Laval. "Preliminary study on suspension plasma sprayed $\mathrm{ZrO}_{2}+8 \mathrm{wt}$. $\%$ $\mathrm{Y}_{2} \mathrm{O}_{3}$ coatings". Journal of the European Ceramic Society. Vol. 31 N $^{\circ} 12$, pp. 20892098. October, 2011. ISSN: 09552219. DOI: 10.1016/j.jeurceramsoc.2011.05.014.

[21] R. Etchart-Salas. "Projection par plasma d'arc de particules submicroniques en suspension. Approche expérimentale et analytique des phénomènes impliqués dans la reproductibilité et la qualité des dépôts". Université de Limoges. 2007.

[22] ASTM E1920-03. "Standard Guide for Metallographic Preparation of Thermal Sprayed Coatings". 2003.

[23] W.C. Oliver and G.M. Pharr. "An improved technique for determining hardness and elastic modulus using load and displacement sensing indentation experiments". Journal of Materials Research. Vol. 7, pp. 1564-1583. 1992.

[24] ASTM E1327-08. "Standard Test Method for Vickers Indentation Hardness of Advanced Ceramics". 2008.

[25] ASTM E1326-08. "Standard Test Method for Knoop Indentation Hardness of Advanced Ceramics". 2003.

[26] R.S. Lima, S.E. Kruger, G. Lamouche and B.R. Marple. "Elastic Modulus Measurements via Laser-Ultrasonic and Knoop Indentation Techniques in Thermally Sprayed Coatings". Journal of Thermal Spray Technology. Vol. 14 $\mathrm{N}^{\circ}$ 1, pp. 52-60. 2005. ISSN: 10599630. DOI: $10.1361 / 10599630522701$.

[27] D.R. Askeland and P.P. Phulé. "Propiedades y comportamiento mecánico". In Ciencia e ingeniería de los materiales. Cuarta edición. José T. Pérez Bonilla and A.A. Gómez Ruíz. Eds., p. 278. 2003.

[28] J. Nohava, R. Mušálek, J. Matějíček and M. Vilémová. "A contribution to understanding the results of instrumented indentation on thermal spray coatings - Case study on $\mathrm{Al}_{2} \mathrm{O}_{3}$ and stainless steel". Surface and Coatings Technology. Vol. 240, pp. 243-249. February, 2014. ISSN: 02578972. DOI: 10.1016/j. surfcoat.2013.12.033.

[29] L. Latka, D. Chicot, A. Cattini, L. Pawlowski and A. Ambroziak. "Modeling of elastic modulus and hardness determination by indentation of porous yttria stabilized zirconia coatings". Surface and Coatings Technology. Vol. 220, pp. 131-139. 2013. ISSN: 02578972. DOI: 10.1016/j.surfcoat.2012.07.025.

[30] P. Carpio, E. Bannier, M.D. Salvador, A. Borrell, R. Moreno and E. Sánchez. "Effect of particle size distribution of suspension feedstock on the microstructure and mechanical properties of suspension 
plasma spraying YSZ coatings". Surface and Coatings Technology. September, 2014. ISSN: 02578972. DOI: 10.1016/j. surfcoat.2014.08.063.

[31] B. Schorr. "Characterization of Thermal Spray Coatings". Materials Characterization. Vol. 42 No 2-3, pp. 93-100. 1999. ISSN: 10445803. DOI: 10.1016/S1044-5803(98)00048-5.
[32] L. Wang, Y. Wang, X.G. Sun, J.Q. He, Z.Y. Pan and C.H. Wang. "Microstructure and indentation mechanical properties of plasma sprayed nano-bimodal and conventional $\mathrm{ZrO}_{2}-8 \mathrm{wt} \% \mathrm{Y}_{2} \mathrm{O}_{3}$ thermal barrier coatings". Vacuum. Vol. $86 \mathrm{~N}^{\circ}$ 8, pp. 1174-1185. February, 2012. ISSN: 0042207X. DOI: 10.1016/j.vacuum.2011.10.029. 